\title{
Educación en derechos humanos. \\ Esbozo de una lectura biopolítica*
}

Artículos temáticos de Educación, N. ${ }^{\circ} 65$ Segundo semestre de 2013 Bogotá, Colombia.

\section{//Human rights education: outline \\ of a biopolitical text}

\section{//Educação em direitos humanos. Esboço de uma leitura biopolitica}

Recibido: 15/07/2013 Evaluado: 08/08/2013

\section{Óscar Orlando Espinel Bernal**}

\begin{abstract}
* Este artículo retoma algunas apuestas, perspectivas metodológicas y elaboraciones del trabajo investigativo recogido en la tesis Una mirada a la educación en derechos humanos en Colombia. Esta investigación fue presentada en la Maestría en Educación de la Universidad Pedagógica Nacional en 2011

Óscar Orlando Espinel Bernal. Docente e Investigador del Departamento de Filosofía de la Corporación Universitaria Minuto de Dios - UNIMINUTO y del Departamento de Posgrados de la Facultad de Educación de la Universidad Pedagógica Nacional. Estudiante del Doctorado en Filosofía de la Universidad de Buenos Aires. Magíster en Educación de la Universidad Pedagógica Nacional, Especialista en Pedagogía del mismo centro universitario y miembro líder del Grupo de Investigación Pensamiento, Filosofía y Sociedad de UNIMINUTO. Correo electrónico: oscar.espinel@yahoo.com
\end{abstract}

\section{Resumen}

Ante la incesante e insistente preocupación desde las instituciones estatales y supraestatales, por promover, expandir y vincular la educación en derechos humanos a los sistemas educativos, se hace necesario tomar distancia y revisar con cierta mirada de sospecha el funcionamiento de las formas político-discursivas en las que se apoyan dichos proyectos dentro del ordenamiento vigente. Para ello nos hemos valido de la noción metodológica foucaultiana de gubernamentalidad, a fin de destacar ciertas regularidades enunciativas que configurarían las prácticas discursivas en torno a la educación en derechos humanos desde el ámbito de la política pública en esta materia.

Regularidades enunciativas como "sistema democrático", "cultura de derechos humanos", "formación para la ciudadanía", "sujeto de derechos" tendrían incidencia, a manera de un macro-nivel -y desde una perspectiva heterárquica del poder-, en las micro-prácticas de las instituciones educativas, siendo posible, tras ellas, examinar formas de subjetivación derivadas del arte de gobernar.

\section{Abstract}

Faced with the incessant and insistent preoccupation on the part of national and supranational institutions to promote, extend and link human rights education with education systems, it is necessary to distance ourselves and review with a certain degree of suspicion the political-discursive ways in which these projects are supported within the current system. In order to do this, in this paper, we have used the Foucauldian methodological notion of governmentality in order to highlight certain recurring practices which configure the discursive practices relating to human rights education from the realm of public policy in this issue, and which have an impact on a macro-level - from a conception of power as heterarchical - in the micro-practices of educational institutions. Revealing practices such as 'democratic system', 'human rights culture', 'citizenship training', 'subject of rights', after which it is possible to examine forms of subjectivation within the power relations derived from the manner of governing.

\section{Palabras Clave}

Educación en derechos humanos, gubernamentalidad, sistemas democráticos, formación de ciudadanos, sujeto de derecho.

\section{Keywords}

Human rights education, governmentality, democratic systems, citizenship training, subject of rights.

\section{Palavras chave}

Educação em direitos humanos, governamentalidade, sistemas democráticos, formação de cidadãos, sujeito de direito. 


\section{Resumo}

Dada a preocupação incessante e insistente das instituições estatais e supra estatais por promover, expandir e vincular a educação em direitos humanos aos sistemas educacionais é preciso tomar distancia e revisar com olhar de suspeita o funcionamento das formas politico-discursivas nas quais se apoiam tais projetos dentro da ordem vigente. Para isso, retomamos a noção metodológica foucaultiana de governamentalidade para salientar algumas regularidades enunciativas que configuram as práticas discursivas ao redor da educação em direitos humanos desde o âmbito da política pública nesta matéria.

Regularidades enunciativas como "sistema democrático", "cultura de direitos humanos", "formação para a cidadania", "sujeito de direitos", teriam incidência, à maneira de um macro-nível -e desde uma perspectiva heterárquica do poder- nas micropráticas das instituições educativas, sendo possível, por trás delas, examinar formas de subjetivação derivadas da arte de governar.

Detenernos con cierta mirada de sospecha y actitud inquisidora en los documentos, planes y proyectos que a nivel local, nacional e internacional propenden a la integración de la educación en derechos humanos (de ahora en adelante EDH) a los sistemas educativos nos permitirá relievar algunos aspectos, intenciones y prácticas de subjetivación que tienden a la producción, control y moldeamiento del tipo de sujetos requeridos dentro de un determinado proyecto de nación que, a su vez, responde o se articula a un proyecto de carácter global.

Estas prácticas de subjetivación, por cuanto operan como técnicas de constitución de sujetos en medio de un entramado de relaciones de poder, se inscriben dentro de las prácticas y saberes gubernamentales ${ }^{1}$. Así, las prácticas y discursos de la $\mathrm{EDH}$ funcionarían dentro de las modalidades gubernamentales de los estados modernos como ejercicio de control sobre las poblaciones a través de la biopolítica. Desde este escenario, los sujetos serían producidos a partir de instancias exteriores de normalización.

Al igual que el concepto de gubernamentalidad, el de biopolítica se constituye como una noción metodológica empleada por Foucault en el desarrollo de su trabajo investigativo en torno al análisis sobre las transformaciones políticas de la modernidad. Se trata, afirma Castro (2008), de "un concepto que debe mostrar su potencialidad analítica en la minuciosidad de los procesos históricos" (p. 191).

1 Castro sintetiza la concepción de gubernamentalidad que Foucault (2009, p. 136) expone en la clase del primero de febrero de 1978 en los siguientes términos: "Foucault entiende por gubernamentalidad: el conjunto constituido por las instituciones, los procedimientos, los análisis y las reflexiones, los cálculos y las tácticas que permiten ejercer el poder sobre la población" (Castro, 2008, p. 190) 
Bajo esta perspectiva, nos unimos en este punto al profesor Noguera, quien plantea lo siguiente respecto a este funcionamiento de la gubernamentalidad como noción metodológica:

Una noción metodológica es una herramienta para pensar, un instrumento para operar sobre un problema. En el sentido particular que quiero señalar en este escrito, una noción metodológica es una herramienta, un instrumento que Foucault elabora para el desarrollo de su actividad de investigación y enseñanza (2009, p. 23).

Y pensar, para Foucault, nos recuerda el profesor Noguera, significa desestabilizar, inquietar, provocar, tensionar, incitar: "para pensar de otro modo, pensar lo impensado antes que conocer o reproducir lo ya sabido" (p. 25).

Es así que en este nivel de la biopolítica y la gubernamentalidad del Estado moderno en cuanto gobierno de la población, de la vida y de las mismas condiciones que puedan favorecer el desarrollo y progreso del sistema político-económico vigente instaurado por el capitalismo globalizado, se configuran las políticas públicas en cuanto perspectiva político-discursiva. En consecuencia, desde esta perspectiva de control biopolítico y de gubernamentalidad como herramientas metodológicas, se hace posible examinar el funcionamiento de enunciados como Estado social de derecho, los valores y principios de derechos de los humanos, valores democráticos, ciudadanía, participación democrática, sujeto de derechos.

Pues bien, esta es la apuesta metodológica del presente trabajo en torno a las prácticas político-discursivas de la educación en derechos humanos que desde esta óptica se presentan como tecnologías que propenden al fortalecimiento y estabilidad del ordenamiento económico y social bajo la postulación de principios universales; sistema que, a su vez, se soporta y legitima, en gran medida, bajo el diseño de las estructuras, sujetos y acomodamientos de los sistemas democráticos vigentes. Afirma Foucault al final de su clase del primero de febrero de 1978: "por lo tanto, el Estado en su supervivencia y el Estado en sus límites solo deben comprenderse sobre la base de las tácticas generales de la gubernamentalidad" (2009, p. 137).

Dentro de esta racionalidad, los derechos humanos se presentan como principios inamovibles, necesarios e incuestionables para la consecución de las aspiraciones generalizadas de paz, convivencia armónica y progreso. Enunciados que resultan vacíos tras su instrumentalización y cooptación al servicio de intereses particulares y que ocultan tras de sí el uso de la población dentro de las lógicas del mercado y la globalización del capitalismo. El código de los derechos humanos se 
convierte así en un discurso que expande y legitima prácticas y estructuras que ocasionan situaciones contrarias a los grandes ideales invocados, pues sus resultados palpables son: injusticia, inequidad, pauperización masiva de la mano de la creciente acumulación y concentración de la riqueza, barbarie e incremento de las diferentes formas de violencia, etc. Cabe anotar que aquí no se intenta rechazar de manera apresurada y acrítica la EDH o el discurso de los derechos humanos en sí mismo. Por el contrario, se trata de pensar de otro modo estos discursos y sus funcionamientos, antes que reproducir lo ya sabido.

En resumen, se trata de prácticas de gobierno en las que el dominio y jurisdicción ya no se ejerce sobre un territorio, sino que el control se ejerce sobre la vida de las personas, sobre la población. En este sentido, Castro (2008) nos aclara que "cuando Foucault habla de la biopolítica, lo hace para referirse al gobierno de ese personaje, no previsto por las teorías filosófico-jurídicas de la soberanía, pero tampoco explicable en términos de ideología, que se llama población" (p. 202). La biopolítica, como ejercicio de análisis de estas diversas formas, tácticas y espacios de gobierno, abre las posibilidades para indagar desde allí las formas de gobierno y las consecuentes formas de subjetivación que operan en y a partir de la educación en derechos humanos.

El presente análisis tiene en cuenta tres grupos de documentos realizados por instituciones supranacionales, como son la Organización de Naciones Unidas (ONU) y el Instituto Interamericano de Derechos Humanos (a partir de ahora IIDH). La razón por la cual el presente estudio se circunscribe a las directrices, lineamientos, concepciones y recomendaciones de estos documentos se relaciona con el hecho de que en medio de la red de relaciones de poder que configura el mundo globalizado actual, los países miembros de la ONU se comprometen a elaborar y ejecutar planes de acción en el campo de la planeación y de las políticas públicas para dar curso y cumplimiento a estos pactos y convenciones internacionales. A partir de una concepción heterárquica del poder $^{2}$, estas formulaciones se presentan

2 En este punto, es necesario precisar, con la ayuda de Castro-Gómez, la perspectiva heterárquica del poder desde la cual se sitúa esta indagación. "Las teorías jerárquicas del poder sostienen que las relaciones más globales de poder estructuran a las menos globales, es decir, que crean las condiciones para que los niveles inferiores queden sometidos a la lógica de los niveles superiores (...) Por el contrario, en una teoría heterárquica del poder (como la que nos ofrece Foucault), la vida social es vista como compuesta de diferentes cadenas de poder, que funcionan con lógicas distintas y que se hallan tan solo parcialmente interconectadas. Entre los diferentes regímenes de poder existen 
como un macronivel del discurso que circula en las instituciones educativas en torno a la $\mathrm{EDH}$, pues su filosofía, orientación, propósitos e intencionalidades, como es de esperar, se vinculan y apropian estas exigencias de la comunidad internacional a la cual está sujeta la nación, a través de mecanismos e instrumentos supranacionales.

\section{El proyecto global de la educación en derechos humanos}

Desde mediados de la década de 1990, los organismos internacionales han dirigido sus esfuerzos hacia los derechos humanos, y especialmente hacia la educación en la esfera de los derechos humanos dentro de la educación formal. Ya en la Conferencia Mundial de Derechos Humanos, realizada en Viena en 1993, se declara la necesidad de iniciar un periodo de tiempo denominado Decenio de las Naciones Unidas para la educación en la esfera de los derechos humanos (19952004). Por su parte, los países integrantes de la Asamblea General de Naciones Unidas se comprometieron a elaborar un plan de acción nacional para la educación en la esfera de los derechos humanos con el fin de unirse a la declaración de dicho decenio. En este proyecto mundial, se mantiene el principio rector que

disyunciones, inconmensurabilidades y asimetrías, de modo que no es posible hablar aquí de una determinación en última instancia por parte de los regímenes más globales (2007, p. 167) afirma que la $\mathrm{EDH}$ es un derecho de los ciudadanos.

En este sentido, en la Conferencia Mundial de Derechos Humanos de Viena (ONU, 1993) se considera que la educación, la capacitación y la información en el campo de los derechos humanos son "indispensables para establecer y promover relaciones estables y armoniosas entre las comunidades y para fomentar la comprensión mutua, la tolerancia y la paz" (citado por ONU, 1996, p. 1). Se entiende que los derechos humanos, en cuanto construcción histórico-social, son esenciales en el mantenimiento del orden social establecido, la armonía y la vida en comunidad. Por esta razón, estos organismos, encargados de mantener y controlar el orden mundial, centran su interés en el fortalecimiento de los derechos al interior de los países y, con ello, la constitución de sujetos para su realización y protección.

Por su parte, en el plan de acción contemplado por la Asamblea General de Naciones Unidas se define la EDH como "el conjunto de actividades de capacitación, difusión e información encaminadas a crear una cultura universal en la esfera de los derechos humanos, actividades que se realizan transmitiendo conocimientos y moldeando actitudes" (ONU, 1996, p. 1). En otros términos, se define como la producción de saberes y prácticas para la expansión de una cultura universal en torno a un código de principios establecidos. Y más adelante, en el plan de acción del Programa 
Mundial para la EDH (ONU, 2006), una vez ampliada esta definición, es posible visibilizar la expresión y cálculo de las formas de subjetivación proyectadas desde la EDH:

Una educación integral en derechos humanos no solo proporciona conocimientos sobre los derechos humanos y los mecanismos para protegerlos, sino que, además, transmite las aptitudes necesarias para promover, defender y aplicar los derechos humanos en la vida cotidiana. La educación en derechos humanos promueve las actitudes y el comportamiento necesarios para que se respeten los derechos humanos de todos los miembros de la sociedad (p. 1).

Así pues, el objetivo principal desde un ejercicio biopolítico es avanzar hacia una cultura universal de derechos humanos en la que el individuo asuma sus responsabilidades y derechos dentro del ordenamiento social establecido. Es decir, promover un escenario universal -con todas las implicaciones de homogeneización y segregación que de ello resulta ${ }^{3}$ - en el cual cada individuo esté en condiciones de vivir en sociedad -en este tipo de sociedad-, respetando y asumiendo una suerte de principios básicos y libertades fundamentales fijadas y brindadas para asegurar-se afirma- la paz, la armonía, el desarrollo y la tolerancia. Es lo que, acudiendo a Foucault, podríamos denominar prácticas gubernamentales que permiten "la supervivencia del Estado" en las sociedades modernas (2009, p. 137).

Al respecto es pertinente recordar con Foucault que a diferencia del poder soberano -previo a constitución de los estados modernos-, donde la ley es el instrumento por excelencia para proteger la soberanía, toda vez que la ley se cumple por la ley misma, en el arte de gobernar desde el que intentamos analizar las formas políticos-discursivas de la EDH, la ley no es necesariamente lo que prima, pues su potencia deviene de su utilidad para alcanzar un fin específico. En otras palabras, en el contexto del poder soberano propio de la época medieval, la ley se identifica

3 Es desde este marco que se inicia el proyecto global de la EDH con el fin de universalizar los principios y valores de los derechos fundamentales y el modelo democrático occidental. Ambos conceptos construidos históricamente, desde y para contexto específicos. "Esta misma tendencia hegemónica rodea el discurso de los derechos humanos y lleva a imponerlos en su universalización desde una perspectiva dogmática" (Espinel, 2011, p. 150). Instauración de una cultura hegemónica que anula la diferencia, la diversidad y la pluralidad. Una cultura universal con pretensiones y expresiones de homogeneización e individualización. 
con la soberanía pues ella -la ley"corresponde a las leyes impuestas por Dios a la naturaleza y a los hombres" (p. 125); en otras palabras, la ley representa el orden establecido por Dios y decretado por el soberano. Por su parte, en el arte de gobernar ejercido en los Estados modernos, la ley obedece a la realización de un fin particular en cuya difusión y adopción incondicional por parte de los ciudadanos, reside la estabilidad y subsistencia del Estado.

Ley y soberanía, entonces, se confundían absolutamente una con otra. Ahora, al contrario, no se trata de imponer una ley a los hombres, se trata de disponer cosas, o sea, de utilizar tácticas y no leyes, o utilizar al máximo las leyes como tácticas; hacer de tal suerte que, por una serie de medios, pueda alcanzarse tal o cual fin (p. 125).

Es en este sentido que se sostiene la primacía de las tácticas y técnicas que hacen posible la realización del fin establecido dentro de las prácticas de gobierno y asumido, de una u otra manera, por los ciudadanos; para el caso del presente análisis, el fin de estas prácticas gubernamentales podría sintetizarse, a nuestro modo de ver, en la expansión, protección y fortalecimiento del modelo democrático moderno occidental, y recientemente, en la consolidación de una democracia de mercado. Allí toman especial relevancia los proyectos de $\mathrm{EDH}$ como instrumento para la instauración de una cultura universal de derechos humanos y de todo un sistema de principios y creencias funcionales para dicho modelo. Prácticas y saberes empleados dentro de las diversas modalidades de las tácticas y técnicas del gobierno de las poblaciones.

Volviendo a la presentación general de los documentos oficiales inspeccionados en este proyecto global liderado por la ONU, el Programa Mundial para la Educación en Derechos Humanos (2005-2009) se inicia como continuación del decenio por parte de la Asamblea General de Naciones Unidas. En este Programa Mundial, se pretende integrar enfáticamente la $\mathrm{EDH}$ al sistema formal de educación primaria y secundaria de las diferentes naciones. Aunque en estos planes de acción mencionados se hace énfasis en los sistemas formales de enseñanza, se tiene claridad en que la EDH debería incluirse en cualquier programa educativo como parte de la formación integral a que tienen derecho los ciudadanos. Con ello se busca garantizar la configuración de una cultura universal de derechos humanos para desde allí, reafirmar las prácticas de gobierno sobre la población.

Otro cuerpo de documentos analizado en este proyecto es el conjunto de informes elaborados por el Instituto Interamericano de Derechos Humanos (IIDH) a propósito del avance en materia de EDH en diecinueve países de la región. 
La EDH es, en estos informes, uno de los campos clave para el análisis de los indicadores de progreso en materia de derechos humanos ${ }^{4}$ en cada país. Es de anotar que, para el momento en que se realizaron los estudios, estos países compartían algunas características en cuanto a sus dinámicas internas e históricas: abandonan un periodo de dictaduras extendido en toda la región e inician la reinstauración de la democracia, así como la consolidación de las instituciones democráticas, la elaboración de reformas constitucionales, legales, administrativas y educativas y la organización y movilización de la sociedad civil para exigir el respeto por los derechos humanos y el Estado de derecho.

Así, la EDH ese presenta como parte fundamental del derecho a la educación. Una educación en la cual se entretejen relaciones de poder y prácticas constitutivas de sujetos y que, por tanto, es capital entre las modalidades específicas de gobierno de las poblaciones. $\mathrm{La} \mathrm{EDH}$, considera el IIDH, es la tarea primordial de las democracias, tanto, que se ha constituido como un derecho que el Estado debe garantizar a sus ciudadanos. Por tanto, impele al Estado entre sus labores de supervivencia ajustar políticas que permitan organizar, estructurar, promover y materializar la práctica de la enseñanza y aprendizaje en el campo de los derechos humanos. "Como derecho, implica la obligación del Estado de asegurar una práctica de la enseñanza y del aprendizaje que incorpore los conocimientos, valores, actitudes y competencias necesarios para formar ciudadanía consciente de ser sujetos de derechos y de responsabilidades" (IIDH, 2003, p. 5). $\mathrm{La} \mathrm{EDH}$, en estos términos, garantizaría o al menos promovería la formación de los individuos en la ciudadanía a partir de los códigos y creencias que se requieren en el funcionamiento del ordenamiento establecido. En definitiva, prácticas de subjetivación en las relaciones de poder dispuestas desde la gubernamentalización del Estado.

Los siguientes apartados se estructuran alrededor de cuatro de los enunciados hallados en la revisión documental, con el fin de trazar en términos generales algunas líneas que permitan apreciar la manera como operaría la EDH en cuanto tecnología para el moldeamiento de los sujetos. Los enunciados en cuestión son: democracia, cultura de derechos humanos, formación de sujetos de derechos y deberes, educación ciudadana. Esta breve

4 Los otros dos campos contemplados en los informes son: acceso a la justicia y participación política. 
indagación nos permitirá apreciar e ilustrar el funcionamiento de la EDH como práctica biopolítica en estas regiones particulares y desde cuatro de los diversos ángulos de análisis posibles.

\section{Una Inversión para la democracia}

En su tercer informe, el IIDH (2004) parece dar un viraje en cuanto al énfasis que da a la EDH. En los dos primeros informes, centraba la atención en la formulación de la EDH como un derecho, haciendo eco a la formulación del artículo 13 del Protocolo de San Salvador (OEA, 1988), y específicamente como elemento esencial y transversal del derecho a la educación. Sin embargo, en su tercer informe, su interés parece estar enfocado hacia la consolidación y fortalecimiento de la democracia a partir de la EDH.

Es por esta razón que el IIDH concibe que la formación de los niños y adolescentes como sujetos de derechos garantizará la estabilidad y el futuro de las democracias, toda vez que estos sujetos serán quienes ejerzan de manera activa la ciudadanía y en dicho ejercicio determinarán el curso de las sociedades.

Según estos documentos analizados, se observa que la EDH, desde una óptica biopolítica, es entendida como la mejor inversión para el establecimiento y mantenimiento de los sistemas democráticos pues ella permitiría, canalizaría, sustentaría y legitimaría el ejercicio del poder del Estado sobre las poblaciones. Un código de valores, principios y creencias que funcionarían, dentro de un proyecto de universalización, como estrategias de adecuación, sometimiento, identificación y compromiso de los ciudadanos con lo que se presenta bajo las figuras de proyecto de nación, progreso, bien común, desarrollo, modernización; solo por nombrar algunas.

En esta instrumentalización de la $\mathrm{EDH}$, es ilustradora la siguiente cita tomada del tercer informe del IIDH (2004):

Confiamos que este III Informe proporcione algunas respuestas $y$, en esa medida, ayude a avanzar hacia la meta de formar niños, niñas y adolescentes conscientes, respetuosos de los derechos humanos y los principios democráticos, y comprometidos con su defensa. La inversión de capital y de recursos pedagógicos en el magisterio de esa edad escolar será, a mediano plazo, la mejor defensa de la democracia en América Latina (p. 6).

La formación en derechos humanos, se dice, fija las condiciones para que el sistema democrático sea viable, confiando en que con ello se estará progresando hacia la paz, la convivencia, el desarrollo sostenible, la inclusión y la justicia social, entre otras promesas. 
Unas líneas antes, este mismo informe nos permite entender las razones que explican porqué vincular la EDH a la escuela y cuál es su propósito en relación con la estabilidad del sistema y el control de la población:

El magisterio que trabaja con niños, niñas y adolescentes es el contingente humano que puede afirmar el rumbo de la democracia y cambiar la conciencia del alumnado que en pocos años estará votando, será miembro de partidos políticos, funcionario público, activista de organizaciones civiles, ciudadano activo y tomador de decisiones (...) Para formarlo integralmente, debemos contar con maestros y maestras de buena calificación científica y técnica y del más alto compromiso humano (p. 6).

Es claro que, si lo que se desea es la estabilidad del sistema democrático, se debe garantizar y controlar los mecanismos de participación en este sistema. Entonces, la principal tarea, se supone, consistiría en formar, modelar, prefigurar los futuros ciudadanos que ejercerán sus derechos, entre ellos su derecho al voto, y, sobre todo, formar o moldear las conciencias que incidirán o padecerán el ordenamiento social establecido. En la escuela, estos futuros ciudadanos no solo están en proceso de formación de su personalidad, sino también de sus subjetividades, ideales, principios y valores. Por esta razón, es vital, en las políticas nacionales e internacionales, fijar los esfuerzos, prácticas, técnicas y formas de subjetivación.

\section{Cultura de derechos humanos}

Ahora bien, continuando con el análisis de las modalidades de subjetivación presentes en el proyecto global de la EDH recopilado en los documentos examinados, emerge otro enunciado que por su regularidad e implicaciones en las prácticas de producción de sujetos merece ser revisado. Se trata del enunciado de cultura de derechos humanos, la cual se inscribe en el proyecto global hegemónico que ha fagocitado el discurso de los derechos humanos.

Como se ha mencionado, con el fin de responder a estas directrices asumidas por las naciones a través de pactos y convenciones internacionales, cada país se ha comprometido a elaborar y ejecutar planes de acción a través de los cuales promover la EDH. En estos planes de acción, se han contemplado los sistemas de educación desde las políticas educativas (reformas institucionales 
y administrativas, leyes y decretos). De igual manera, se ha proyectado incidir en los planes de estudio, contenidos, libros de texto ${ }^{5} y$, en general, en los currículos de las instituciones educativas. Todos estos esfuerzos se plantean con la intención de integrar en la escuela la educación, capacitación e información en derechos humanos que posibiliten la tan anhelada cultura universal de derechos humanos.

Todo lo que se enseña, y la manera como se enseña, debe manifestar los valores y principios de los derechos humanos y propiciar la formación de sujetos de derecho comprometidos con la vigencia y ejercicio efectivo tanto de sus derechos como los de sus semejantes.

Para ello, es esencial asegurar que la enseñanza y el aprendizaje de los derechos humanos tengan lugar en un entorno de aprendizaje basado en los derechos y que los objetivos de la educación, las prácticas y la organización de las escuelas sean compatibles con los valores y principios de los mismos. Asimismo, es importante que esos

5 En este punto, es interesante y bastante gráfico resaltar la manera como estas estrategias de biopolítica orquestadas desde las políticas públicas encuentran un aliado imprescindible y potente en los mercados y, en particular, en los mercados del libro de texto y de la producción de material escolar. Allí no solo se regula y codifica lo que ha de enseñarse, sino cómo ha de enseñarse. principios se arraiguen en la cultura de la escuela, en la comunidad escolar y en la comunidad más amplia que la rodea (ONU, 2006, p. 50).

La EDH como parte constitutiva del derecho a la educación debe entenderse, de acuerdo con los proyectos globales y sus adecuaciones en los planes nacionales, desde una perspectiva transversal, dentro y fuera de la escuela. Con ello se busca garantizar su vigencia tanto en la enseñanza formal como informal. Esta perspectiva transversal se presenta como un elemento inclusivo de una cultura de derechos humanos. La definición y los contenidos de la EDH han ido creciendo dentro de un proceso progresivo a partir de su incorporación en el sistema universal de los derechos humanos. Esta configuración se ha visto apoyada y fortalecida por los diferentes instrumentos internacionales y por su implementación en las diversas constituciones políticas y legislaciones nacionales, así como por los planes de acción y programas de los países para dar cumplimiento a sus compromisos con la comunidad internacional.

Cabe mencionar, además, la recomendación del IIDH (2006) en su quinto informe en relación con que todas las adaptaciones y contextualizaciones adelantadas en los diferentes niveles (en nuestro caso, escuela y docentes) deben ajustarse a la normatividad y lineamientos 
fijados por las entidades superiores, pues esto garantiza el control y la supervisión de los procesos.

Los modelos más centralizados tienden a disminuir la complejidad de los procedimientos, al costo de reducir la participación de los actores y de sus opiniones; otros modelos ponen el énfasis en los extremos nacional y local colocando las competencias para lo normativo y técnico en el primero y la obligación de interactuar con la comunidad educativa en el segundo (la institución educativa local) (p. 39).

En otras palabras, al Estado le corresponde generar la políticas y lineamientos para desarrollar e integrar la EDH, y a las instituciones y educadores les compete ejecutarlas y velar por su cumplimiento. Es algo paradójico si recordamos que el propósito final es la generación y promoción de ambientes democráticos porque la creatividad, innovación, idoneidad, adaptación, contextualización y creación propia de los sujetos se limita a la obediencia y funcionamiento de los mecanismos de participación centralizados, normalizados, controlados y filtrados por los niveles superiores en un sistema jerárquico.

\section{Formación de sujetos de derechos y deberes}

Otro elemento que se pone de manifiesto en el análisis biopolítico realizado sobre los propósitos de estos programas es la recurrencia en la preocupación por la formación de los sujetos de derechos a través de los discursos y prácticas de EDH. La finalidad fundamental que sostiene las pretensiones de los programas de $\mathrm{EDH}$ es que cada individuo se reconozca, precisamente, como sujeto de derechos. Y reconocerse como sujeto de derechos es estar en capacidad de ejercerlos y exigir respeto hacia ellos, pero también, reconocerlos en el otro y de igual manera, respetarlos y promoverlos. Reconocer derechos, un sistema de derechos, desde la óptica en cuestión, implica, simultáneamente, reconocer deberes. Un sujeto de derechos y deberes funcional en las prácticas gubernamentales analizadas.

Así, el Plan Nacional de Educación en Derechos Humanos (a partir de ahora PLANEDH) inicia su introducción con las siguientes palabras:

Sin duda la EDH es la vía más racional y prometedora para aprender y tomar conciencia acerca de los valores y 
principios que enaltecen la dignidad del ser humano, destacar la importancia que han adquirido para regular de mejor forma las relaciones del Estado y la sociedad y para comprender de qué manera los derechos humanos se han transformado en una fuente de inspiración para construir respuestas adecuadas a la creciente complejidad sociopolítica, a los continuos cambios sociales, a las nuevas demandas que se exigen al sistema educativo y determinar las responsabilidades que corresponden al Estado en este escenario (MEN, 2007, p. 4).

Este es el primer párrafo de la Introducción del PLANEDH, donde se presenta la EDH como instrumento para reconocer, promocionar y afianzar los valores de la sociedad (occidental) y así regular -como ejercicio de poder y dominio- las relaciones entre el Estado y la sociedad y viceversa. Se entiende así a los derechos humanos como el código que establece las reglas de juego, que legitima las acciones y relaciones entre uno y otro, que pone límites y senderos al ejercicio de la libertad, a las aspiraciones y proyecciones personales, a la misma dignidad humana por cuanto ya está intrínsecamente definida por el sistema de principios y valores que la enaltecen $y$, obviamente, regula las exigencias personales frente al Estado y del Estado frente al sujeto. Un sujeto de derecho.

Ahora bien, la ampliación progresiva del alcance del concepto de derechos humanos y de su integralidad e interdependencia ha enriquecido esta finalidad definida a partir del sujeto, complejizando así el papel de la educación en derechos humanos en cuanto que, además de operar como un instrumento de prevención de violaciones de los derechos fundamentales, debe constituirse en tanto formas de subjetivación, en un motor de transformaciones individuales y sociales, de construcción de ciudadanía y de realización de la democracia (IIDH, 2006, p. 12). No se limita entonces a la prevención y eventual denuncia de la violación de derechos humanos, sino que avanza al ejercicio de iniciativas y condiciones transformadoras de las sociedades en el marco establecido de la ciudadanía y la vida democrática. Constituyéndose así en los límites para el ejercicio transformador a nivel individual y social.

\section{Educación ciudadana}

En los últimos años, hemos presenciado un auge creciente del discurso de la ciudadanía y formación para la ciudadanía en gran parte de los escenarios de la sociedad, pero principalmente en el campo de las ciencias sociales y, de manera particular, en la educación. Hay un incremento progresivo en el interés de 
los distintos sectores del Estado por consolidar unas competencias que garanticen la correcta formación de los ciudadanos que componen la sociedad. La recurrencia de este discurso responde a las características y acontecimientos de una época convulsionada que hereda algunas secuelas del pasado reciente. Ruiz y Chaux (2005) citan a Will Kymlicka (2001) al enumerar estas situaciones que han venido debilitando o evidencian un debilitamiento de las estructuras e instituciones del ordenamiento democrático, entre las cuales podemos mencionar:

La creciente abstención electoral en las sociedades democráticas, el resurgimiento de movimientos nacionalistas en algunos países económicamente desarrollados, las tensiones sociales propias de una población recientemente multirracial y multicultural, la crisis del Estado de Bienestar y el desfavorable balance que hasta ahora arrojan las políticas medioambientales, entre otros (p. 10).

Todos estos son acontecimientos políticos y sociales que marcan el escenario político contemporáneo y que se convierten en una gran preocupación para la armonía y perdurabilidad del sistema democrático, a los cuales debe sumarse el permanente ambiente de guerra presente en el mundo a lo largo de todo el siglo XX y que continúa, con vehemencia, hasta nuestros días.

En el escenario colombiano, la situación no es diferente; por el contrario, parece intensificarse debido al conflicto interno que ha padecido nuestra sociedad de manera más aguda en la segunda mitad del siglo XX, aunque no podemos desconocer que estos conflictos tienen sus raíces y manifestaciones mucho más atrás en la historia de nuestro país. Este conflicto ha conducido a la sociedad colombiana, dicen Ruiz y Chaux (2005), a una crisis de humanidad y "dicha crisis también ha significado una crisis de ciudadanía, esto es, de la posibilidad de construir condiciones sociales justas y equitativas para todos articuladas por la vía política" (p. 10). Es precisamente en este contexto en el cual surge el Plan Nacional de Educación en Derechos Humanos (PLANEDH).

Esta convulsionada realidad inevitablemente afecta el proyecto político democrático establecido en la nueva Constitución Política de 1991, en la que se presenta al Estado colombiano como un Estado social de derecho en el cual, además de proteger, promover y garantizar los derechos civiles y políticos, el Estado se compromete con el reconocimiento de los 
derechos económicos, sociales y culturales. Esta Constitución "provee las orientaciones, mecanismos y procedimientos requeridos para la construcción no solo de un sistema democrático, sino, lo que es más importante, de una cultura de la democracia" (p. 11). Proyecto que se ve en peligro ante los acontecimientos enunciados, los cuales van deteriorando la consistencia de las estructuras democráticas del país y van incrementando la pérdida generalizada de confianza en sus instituciones y representantes. Todo ello atentando contra la estabilidad del Estado y el gobierno que ejerce sobre la población.

El concepto de Estado social de derecho se desarrolla en tres principios orgánicos: legalidad, independencia y colaboración de las ramas del poder público para el cumplimiento de los fines esenciales del Estado y criterios de excelencia. El respeto por los derechos humanos y el acatamiento de unos principios rectores de la actuación estatal constituyen las consecuencias prácticas de la filosofía del Estado Social de Derecho. De esta manera, un Estado Social de Derecho se consolida cuando tanto el ciudadano como el Estado comparten valores $y$ actitudes de naturaleza universal (PLANEDH, 2007, p. 61).

Kymlicka insiste en que la educación de la ciudadanía ${ }^{6}$ es una de las funciones esenciales de las instituciones educativas, y que de hecho este fue uno de los fines con los cuales fue creado todo un sistema educativo. "Uno de los cometidos básicos de la escuela consiste en preparar a las próximas generaciones para sus responsabilidades como ciudadanos" (Kymlicka, 2001, p. 251). Sin embargo, en la EDH operando como táctica de biopoder, se trata de ciudadanos que estén en la capacidad, y sobre todo en la disposición, de conservar las estructuras sociales dentro de las cuales y para las cuales están y han sido moldeados a lo largo de su vida. "Lo que implica que además de las reglas y las prácticas establecidas para el ejercicio del gobierno, se requiere de una subjetividad y una cultura en las que la democracia se viva como conceptos, valores y hábitos", tal como afirman Los Lineamientos

6 "La ciudadanía es la condición política que nos permite participar en la definición de nuestro propio destino, es algo que o bien se acata o bien se ejerce. (...) El acatamiento de la ciudadanía implica una comprensión básica de las costumbres, valores, tradiciones, formas de interacción e intercambio simbólico del lugar que habitamos. Ello a la vez constituye el fundamento de la civilidad. (...) Ser ciudadano activo, por otra parte, significa ejercer con sentido de responsabilidad un rol político, que, en buena medida, se define en la participación de proyectos colectivos en los que se hace tangible la idea de la construcción o reconstrucción de un orden social justo e incluyente" (Ruiz y Chaux, 2005, pp. 15-16). 
curriculares para formación en la constitución política y la democracia (MEN, 1998a, p. 13) y en clara articulación con las prácticas gubernamentales, el ejercicio biopolítico y las modalidades de subjetivación orquestadas desde la política pública en la esfera de la EDH. Por su parte, y reafirmando lo anterior, en la formación de competencias ciudadanas se asume que "aquello a lo que políticamente aspira una sociedad y que se encuentra plasmado en su Constitución no puede ser excluido de sus prácticas educativas, por ello resulta tan importante la formación ciudadana" (Ruíz y Chaux, 2005. p. 11). Una formación de ciudadanía articulada a prácticas biopolíticas que, en cuanto moldeamiento de subjetivades, creencias y aspiraciones, opera como tecnología de control de la población.

Como es de esperar, de acuerdo con lo que hemos venido exponiendo, esta educación para la ciudadanía va más allá de una educación cívica y el reconocimiento de leyes e instituciones:

La educación ciudadana no consiste únicamente en aprender los elementos básicos de las instituciones y los procedimientos de la vida política. También implica la adquisición de una serie de disposiciones, virtudes y lealtades que están íntimamente ligadas con la práctica de la ciudadanía democrática (Kymlicka, 2001, p. 251).

Por tanto, la educación para la ciudadanía no se reduce al cumplimiento obediente y pasivo de las normas; además de ello, se amplía a la consolidación de una voluntad individual y colectiva que esté dispuesta a aceptar dicho ordenamiento, pero también a comprometerse y procurar, en cada una de sus acciones, instaurar y conservar tal ordenamiento. Este es el objetivo del biopoder ejercido por el Estado sobre cada uno de los integrantes de la sociedad para su conducción y gobierno. Y en relación con ello, de manera especial, luego de su interiorización como práctica de sí, esta formación debe conducir al ciudadano a rechazar todas aquellas acciones y conductas que puedan atentar contra la sociedad. Con lo cual, el ciudadano se une a las acciones policivas de vigilar y controlar los comportamientos de los demás conciudadanos. El ciudadano, además de reconocer y respetar desde sus acciones el ordenamiento social, se convierte, a partir de la educación para la ciudadanía, en garante y protector del sistema social y político.

Y esto es precisamente lo que busca la propuesta hecha desde los Lineamentos curriculares del MEN para ética y valores (MEN, 
1998b) en torno a la formación de competencias ciudadanas: el que a partir de las instituciones educativas sea posible no solo identificar lo que significa ser ciudadano, sino también promover conocimientos y habilidades a partir de las cuales la comunidad que interviene y participa del proceso formativo se involucre, comprometa y trabaje por la realización del proyecto de nación que se presenta en la Constitución.

\section{Una reflexión final: superar la categoría de sujeto de derechos}

Los derechos humanos como carta de navegación, reguladores y controladores de las prácticas sociales y de las relaciones entre los agentes sociales, instituciones y organizaciones, legitiman y ofrecen el marco de referencia en el que se instituye lo legal o ilegal, lo correcto e incorrecto, lo viable e inviable. En este horizonte, es posible caer en cierta percepción a-histórica del cuerpo de derechos, la cual los sitúa como principios universales, fijos, inamovibles, naturales y, por tanto, incuestionables. Este a-historicismo ignora e invisibiliza la condición social de los derechos humanos y la historia de su formación, instituyéndolos como principios abstractos, dogmáticos, formales e instrumentales.

Así, los derechos humanos, como es sabido, se esgrimen como la base para la consolidación de la pretendida libertad, justicia y solidaridad en los Estados modernos autodefinidos como democráticos, pero que usualmente, distan de ello y tienden a asemejarse a sistemas autoritarios camuflados. Libertad, justicia, solidaridad y democracia entendidas y promulgadas desde el marco del sistema capitalista imperante. Libertad restringida y cooptada; justicia administrada desde intereses específicos; solidaridad centrada en la individualidad y democracia legitimadora de prácticas que frecuentemente se inclinan más a sistemas autoritarios y oligárquicos.

Lo que estoy queriendo poner en evidencia desde esta perspectiva político-discursiva en torno a la EDH y con ayuda de las nociones metodológicas de gubernamentalidad y biopolítica es una suerte de círculo vicioso en el cual la acción del Estado, en manos de sus gobernantes, se rige por una serie de principios, valores y derechos que él mismo establece y regula. Es decir, el mismo sistema democrático expandido bajo el modelo occidental, establece la normatividad y regulación para su actuar y con ello, los principios que lo controlan y legitiman. En consecuencia, desde la óptica aquí propuesta, la EDH promovida por la política pública se limita al reconocimiento del articulado y de los mecanismos para su protección.

La transformación recurrentemente citada en los discursos de la política pública se encuentra regulada por el sistema universal de derechos humanos. Cualquier acción que se salga de dichos parámetros socialmente aceptados, puede ser sancionada 
y repudiada. El cuerpo de los derechos es el sendero por donde es posible transitar; es el terreno y los límites; establece los puntos cardinales, el norte para la acción social; determina y orienta las conductas, formas de vida de las personas y las relaciones entre el Estado y sus ciudadanos y viceversa. En síntesis, se instituye la reivindicación de un modelo ya fijado a partir de la autorregulación propia de la reafirmación de los sujetos de derechos.

De esta manera, la transformación en el marco de los derechos humanos así entendidos es, en definitiva, el mantenimiento, o, si se quiere, el mejoramiento del orden establecido por el sistema económico y social actual, que paradójicamente es fuente de desigualdad social, marginación, injusticia e inequidad social, pauperización de la población y violencia. Es, en últimas, la reacomodación del sistema para mantener el mismo estado de cosas bajo las promesas de justicia, libertad y paz que el mismo sistema anula en su fortalecimiento y expansión.

Se impulsa la EDH cimentada en estas esperanzas en un mundo mejor, justo y en paz. Pero esto, desde la institucionalidad, las políticas públicas indagadas y las democracias modernas capitalistas, se queda en falsas promesas y, por tanto, en falsas esperanzas. A pesar de ello, surten su efecto en el fortalecimiento de las instituciones y valores del ordenamiento vigente en las prácticas gubernamentales como ejercicio biopolítico de control de la población.

Estos enunciados no serán más que palabras vacías hasta que la formación en cuestión no supere la categoría de sujetos de derechos inmersos en un sistema rígido y universalista de derechos que se presenta como fundamento y discurso legitimador de ese mismo ordenamiento sociopolítico regido por el sistema económico que oprime, subyuga, violenta y margina en la medida que acumula y concentra las riquezas y privilegios en unos pocos. Privilegios entre los que se cuentan los mismos derechos. Derecho a la libertad, a la educación, a la salud, a la vida, al trabajo, al libre desarrollo de la personalidad, a la dignidad humana, etc.

Es necesario, entonces, hallar los caminos que permitan que los derechos humanos, en cuanto construcción social, histórica y política, se constituyan en auténticas herramientas de empoderamiento, transformación y creación de nuevas alternativas dentro de las comunidades. Tarea en la que ha pretendido contribuir esta indagación a fin de desnaturalizar, deconstruir, problematizar y complejizar un discurso universal, universalizante e instrumentalizado, que ha vaciado la potencia de los 
interrogantes y apuestas que, en nuestra opinión, lleva consigo la idea de derechos humanos.

\section{Referencias bibliográficas}

Castro, E. (2008). Biopolítica: de la soberanía al gobierno. Revista Latinoamericana de Filosofía, 2( XXXIV), 187-205.

Castro-Gómez, S. (2007). Michel Foucault y la colonialidad del poder. Revista Tabula Rasa, 6.

Departamento Nacional de Planeación, DNP. (2007). Plan Nacional de Desarrollo 2006-2010. Estado comunitario: desarroIlo para todos. Recuperado de http://www.dnp.gov.co/PortaIWeb/LinkClick.aspx?fileticket= WSgQTUkodjQ\%3d\&tabid=65

Espinel Bernal, O. (2011). Una mirada a la educación en derechos humanos en Colombia. (Tesis de Maestría en Educación). Universidad Pedagógica Nacional, Bogotá.

Foucault, M. (2009). Seguridad, territorio, población. Buenos Aires: Fondo de Cultura Económica.

Gallardo, H. (2006). Derechos humanos como movimiento social. Bogotá: Ediciones Desde Abajo. Instituto Interamericano de Derechos Humanos. (2002). I Informe Interamericano de la Educación en Derechos Humanos. Un estudio en 19 países. Parte I: DesarroIlo Normativo. Recuperado de: http://www.iidh.ed.cr/BibliotecaWeb/PaginaExterna.aspx?url=/
BibliotecaWeb/Varios/Documentos/BD_632226527/Informe I - espanol.pdf

Instituto Interamericano de Derechos Humanos. (2003). II Informe Interamericano de la Educación en Derechos Humanos. Un estudio en 19 países. Desarrollo en el currículo y textos escolares. Recuperado de: http:// www.iidh.ed.cr/BibliotecaWeb/ PaginaExterna.aspx?url=/BibliotecaWeb/Varios/Documentos/ BD_632226527/Informe II - espanol.pdf

Instituto Interamericano de Derechos Humanos. (2004). III Informe Interamericano de la Educación en Derechos Humanos. Un estudio en 19 países. Desarrollo en la formación de educadores. Recuperado de: http://www.iidh.ed.cr/ BibliotecaWeb/PaginaExterna. aspx?url=/BibliotecaWeb/Varios/ Documentos/BD_632226527/ Informe III - EDH.pdf

Instituto Interamericano de Derechos Humanos. (2005). IV Informe Interamericano de la Educación en Derechos Humanos. Un estudio en 19 países. Desarrollo en la Planificación Nacional. San José. Recuperado de: http:// www.iidh.ed.cr/BibliotecaWeb/ PaginaExterna.aspx?url=/BibliotecaWeb/Varios/Documentos/ BD_632226527/Informe IV EDH.pdf

Instituto Interamericano de Derechos Humanos. (2006). V Informe Interamericano de la Educación en Derechos Humanos. Un estudio 
en 19 países. Desarrollo en los contenidos y espacios curriculares: 10-14 años. Recuperado de: http://www.iidh.ed.cr/BibliotecaWeb/PaginaExterna.aspx?url=/BibliotecaWeb/Varios/ Documentos/BD_438003671/Informe V - EDH.pdf

Instituto Interamericano de Derechos Humanos. (2007). VI Informe Interamericano de la Educación en Derechos Humanos. Un estudio en 19 países. Desarrollo normativo de la educación en derechos y el gobierno estudiantil. Segunda medición. Recuperado de: http://www.iidh.ed.cr/

Kymlicka, W. (2001). Educación para la ciudadanía. En Colom, F. (ed.), El espejo, el mosaico y el crisol. Modelos políticos para el multiculturalismo (251-283). Barcelona: Anthropos.

Magendzo, A. (2005). Educación en derechos humanos. Bogotá: Editorial Magisterio.

Ministerio de Educación Nacional (MEN) (1998a). Serie Lineamientos Curriculares. Formación en la Constitución Política y la Democracia. Recuperado de: http://www.mineducacion.gov.co/cvn/1665/articles-89869_archivo_pdf6.pdf

Ministerio de Educación Nacional (MEN) (1998b). Serie Lineamientos Curriculares. Educación ética y Valores humanos. Recuperado de: http://www.mineducacion.gov.co/ cvn/1665/articles-89869_archivo_pdf7.pdf

Ministerio de Educación Nacional (MEN), Defensoría del Pueblo, Programa Presidencial para los derechos humanos y el derecho internacional humanitario, con la asistencia de la Oficina del Alto Comisionado de las Naciones Unidas para los Derechos Humanos. (2007). Plan Nacional de Educación, Respeto y Práctica de los Derechos Humanos, PLANE$D H$. Colombia.

Noguera, C. (2009). La gubernamentalidad en los cursos del profesor Foucault. Revista Educaçao e Realidade 2(34), 21-34.

Organización de Estados Americanos (OEA) (1988). Protocolo adicional a la convención americana sobre derechos humanos en materia de derechos económicos, sociales y culturales, Protocolo de San Salvador. Recuperado de: http://www. cidh.oas.org/Basicos/basicos4.htm

Organización de Naciones Unidas (ONU) (1993). Conferencia Mundial de Derechos Humanos. Declaración y Programa de Acción de Viena. Recuperado de: http://www.unhchr.ch/ huridocda/huridoca.nsf/(Symbol)/A.CONF.157.23.Sp

Organización de Naciones Unidas (ONU) (1996). Cuestiones relativas a los derechos humanos: cuestiones relativas a los 
derechos humanos, incluidos distintos criterios para mejorar el goce efectivo de los derechos humanos y las libertades fundamentales. Recuperado de: http:// www.unhchr.ch/huridocda/huridoca.nsf/(Symbol)/A.51.506. Add.1.Sp

Organización de Naciones Unidas (ONU) (2006). Plan de Acción
Programa Mundial para la EDH. Primera etapa (2005-2007). Recuperado de: http://www.ohchr. org/Documents/Publications/ PActionEducationsp.pdf

Ruiz Silva, A. y Chaux Torres, E. (2005). La formación de competencias ciudadanas. Bogotá: Ascofade. 
